\title{
ILCEA
}

Revue de l'Institut des langues et cultures

d'Europe, Amérique, Afrique, Asie et Australie

43 | 2021

Images des Amériques : fabrique, représentations, usages

\section{Noticiero ICAIC : les images des Amériques dans les actualités cinématographiques cubaines (1960-1990)}

Noticiero ICAIC: Images of the Americas in Cuban Newsreel (1960-1990)

\section{Camila Arêas}

\section{OpenEdition}

\section{Journals}

Édition électronique

URL : https://journals.openedition.org/ilcea/13389

DOI : $10.4000 /$ ilcea. 13389

ISSN : 2101-0609

Éditeur

UGA Éditions/Université Grenoble Alpes

Édition imprimée

ISBN : 978-2-37747-299-4

ISSN : $1639-6073$

Référence électronique

Camila Arêas, « Noticiero ICAIC : les images des Amériques dans les actualités cinématographiques cubaines (1960-1990) », ILCEA [En ligne], 43 | 2021, mis en ligne le 30 juin 2021, consulté le 30 juin 2021. URL : http://journals.openedition.org/ilcea/13389; DOI : https://doi.org/10.4000/ilcea.13389

Ce document a été généré automatiquement le 30 juin 2021

(C) ILCEA 


\title{
Noticiero ICAIC : les images des Amériques dans les actualités cinématographiques cubaines (1960-1990)
}

\author{
Noticiero ICAIC: Images of the Americas in Cuban Newsreel (1960-1990)
}

\section{Camila Arêas}

\section{Introduction}

\begin{abstract}
La révolution cubaine a constitué, pour le cinéma, une importante source d'images d'archive. On peut dire que le cinéma révolutionnaire latino-américain trouvera dans les archives de l'ICAIC du matériel pour les aider à reconstruire une véritable histoire pour leur peuple. [...] Pour nous, cinéastes cubains, aborder l'Histoire, la raconter dans sa juste mesure, l'interpréter n'est pas seulement une opération cinématographique, mais a été et continuera d'être une nécessité idéologique, culturelle et politique. Renoncer à cette ligne d'action, c'est renoncer au combat, c'est renoncer aux armes et aux outils de travail dont nous disposons aujourd'hui au sein d'une confrontation idéologique incontournable. (Santiago Álvarez, dans Aray, $1983: 12,13)$
\end{abstract}

Journal d'actualités cinématographiques produit entre 1960 et 1990 par les cinéastes de l'Institut cubain des arts et de l'industrie cinématographiques (ICAIC) et diffusé toutes les semaines dans les salles de cinéma de l'île, le Noticiero était ainsi défini par son directeur, Santiago Álvarez, comme une source d'images d'archive capables d'intervenir dans le cours de l'histoire cubaine, latino-américaine et mondiale. Le cinéjournal critique et internationaliste a été conçu par Fidel Castro et son ami proche devenu le directeur de l'ICAIC, Alfredo Guevara, un an après le triomphe de la révolution dans le but d'enregistrer le processus révolutionnaire qu'ils voulaient voir s'étendre dans les pays latino-américains et "tiers-mondistes» pris dans des luttes anti-impérialistes et postcoloniales. Les enjeux idéologiques sous-jacents à la 
production du Noticiero, dans un contexte marqué par la censure médiatique régionale, nous conduisent à placer les représentations véhiculées par ce ciné-journal au centre d'une problématique sémiotique qui cherche à saisir le sens des images par rapport à la stratégie politique cubaine.

2 Après 30 ans d'oubli, ces archives ont été récemment restaurées par l'Institut national de l'audiovisuel (INA), en France, et réapparaissent aujourd'hui dans l'espace public, ouvrant une série des questions au sujet des représentations des Amériques dans une perspective continentale et dans le contexte de la Guerre froide. Issu d'une recherche en cours ${ }^{1}$ en sciences de l'information et de la communication, cet article développe une analyse sémiotique avec l'objectif de rendre visibles et lisibles les dimensions matérielles, symboliques et pragmatiques des images et représentations des Amériques véhiculées par le Noticiero.

Quelles images configurent le récit ciné-journalistique du Noticiero dans la couverture des actualités d'Amérique latine et des États-Unis? Quelles ont été leurs conditions de production et de circulation (matérielles, techniques, géopolitiques) ? Quelles ont été leurs visées rhétoriques, énonciatives et performatives? Comment ces images ont été au service d'une stratégie politique et révolutionnaire?

Pour répondre à cette problématique, nous nous pencherons sur l'ensemble des émissions du Noticiero couvrant les actualités nord et latino-américaines afin de développer une analyse sémiotique des images et des commentaires qui les accompagnent. Entre la première (6 juin 1960) et la dernière ( 7 juillet 1990) édition du Noticiero, nous comptons un total de 1492 émissions produites. Sur l'ensemble de cette production, le corpus étudié ici intègre seulement les émissions sur les États-Unis (107), le Brésil (23), le Chili (27), l'Argentine (9), l'Uruguay (13) et la Bolivie (11) ${ }^{2}$. Dans le cas des pays latino-américains, nous avons constitué deux sous-corpus d'émissions correspondant respectivement aux périodes antérieure et postérieure aux coups d'État militaires, lesquels inaugurent les « années de plomb » en Amérique latine.

Pour traiter ce corpus selon une approche d'analyse sémiotique, nous nous appuierons sur les théories et méthodes en SIC propres au domaine de l'analyse des images et des récits médiatiques (Maria Giulia Dondero, Marc Lits, Umberto Eco, Oswald Ducrot), ainsi que de la pragmatique du langage (Jocelyne Arquembourg, John Austin, Charles Peirce Sanders) afin de saisir les propriétés saillantes du langage expérimental qui fait la marque du Noticiero. En nous focalisant sur l'analyse des images et de leur narration à partir d'une interrogation sur le concept de "démonstration " (Arquembourg, 2005, 2010), nous chercherons à dessiner une typologie des propriétés - rhétoriques, énonciatives, performatives - qui nous permettent d'analyser la relation des "images-commentaires" du point de vue de leurs caractéristiques langagières et de leurs visées pragmatiques. Pour ce faire, nous avons choisi, sur l'ensemble du corpus, les séquences les plus significatives au regard de notre problématique, tout en respectant la représentativité de chaque pays. Au moyen de cette approche sémiotique et en nous appuyant sur des entretiens réalisés avec les cinéastes de l'ICAIC ${ }^{3}$, cet article a pour objectif de comprendre le rôle primordial joué par les images fixes (photographies, cartographies, infographies) et les images en mouvement (séquences filmées, images d'animation et d'archive) dans la configuration $\mathrm{du}$ récit cinématographique cubain sur les actualités en Amérique latine et aux États-Unis entre 1960 et 1990. 


\section{Les actualités américaines : agenda, cadrage et conditions de production dans le contexte de la Guerre froide}

6 L'étude des représentations des Amériques dans le Noticiero nous amène tout d'abord à saisir le répertoire des langages qui configurent l'« agenda ${ }^{4}$ » (McCombs \& Shaw, 1972), le « récit » (List, 2008) et le " cadrage » (Entman, 1993) ${ }^{5}$ ciné-journalistique cubains sur les actualités continentales en contexte de Guerre froide. La cartographie (quantitative et qualitative) des représentations des actualités nord et latino-américaines que nous dessinons ici est indissociable de l'étude des conditions de production et de circulation des images véhiculées par le Noticiero. Cette double analyse nous permettra de répondre aux deux premières questions posées dans cet article afin de démontrer comment la conjoncture géopolitique continentale a radicalement changé le langage et redéfini le rôle joué par les images dans les représentations des Amériques au long de 30 ans de diffusion des actualités cinématographique cubaines.

\subsection{Un ciné-journal fait pour et par l'Amérique latine : un agenda contre l'ennemi « d'en face »}

7 La première sortie du Noticiero a eu lieu le 6 juin 1960 durant la Guerre froide qui divisait alors le monde en termes de «zones d'influence» états-uniennes et soviétiques ${ }^{6}$. Sur les écrans de cinéma de La Havane, le continent américain était représenté comme la scène par excellence de cette géopolitique et les États-Unis, comme «l'ennemi d'en face » (Labrada, en entretien) qu'il fallait renverser ou, alors, comme la "machine de guerre » capitaliste qu'il fallait contenir dans ses pulsions impérialistes. Entre 1960 et 1990, nous comptabilisons 107 éditions du Noticiero couvrant les actualités nord-américaines. Dans la couverture nationale, le regard cubain met la focale sur les mouvements afro-américains pour les droits civiques (11 éditions) et les manifestations populaires contre la guerre au Vietnam (9 éditions). Dans la couverture internationale, c'est notamment la bataille nucléaire, la course à l'armement, la guerre des étoiles et, encore plus important, les invasions au Vietnam (193 éditions), en Corée et en Afghanistan qui façonnent le cadrage cubain des actualités nord-américaines.

8 En termes qualitatifs, les images et discours qui configurent le macro-récit cubain sont toujours défavorables aux États-Unis au profit des avancées, conquêtes et progrès observés dans l'Union soviétique et les pays satellites du bloc communiste. D'un côté, les mésaventures nord-américaines en Asie et de ses représentants à l'ONU et à l'OEA sont racontées sous le ton de l'humour, du sarcasme et de la dénonciation, selon un paradigme discursif qui cherche à renverser la domination. De l'autre, les réussites soviétiques dans l'éducation, la culture, le sport et l'industrie sont promues comme les signes d'une nouvelle souveraineté communiste, selon un paradigme discursif de type propagandiste.

Dès 1960, à la suite du triomphe de la révolution, la guerre idéologique menée par Cuba trouvait dans le Noticiero "une arme de combat, un moyen de contre-information, d'agitation et de propagande en faveur de la libération des pays du tiers monde " 
(Amiot-Guillouet, 2008, dans Aray, 1983). Dans la conception de Santiago Álvarez et des cinéastes de l'ICAIC, le terme de «tiers-monde » renvoie alors aux pays du Sud - latins, africains et asiatiques - pris dans des guerres anticoloniales ou anti-impérialistes ${ }^{7}$. Comme le décrit le directeur de l'ICAIC, Alfredo Guevara: "Ce ne sont pas des actualités uniquement pour Cuba, la révolution cubaine est internationaliste et solidaire. [...] L'internationalisme du Noticiero n'était pas seulement pour l'Amérique latine, il était tiers-mondiste. » (Álvarez, $2012: 56,57$ )

10 Néanmoins, l'agenda international du Noticiero se tournait davantage vers les pays voisins, les "frères" latino-américains qu'il fallait libérer au moyen d'un langage expérimental propre au "cinéma urgent » de Santiago Álvarez. En effet, nous notons que la couverture latino-américaine précède toujours l'actualité internationale dans l'ordre du montage. L'actualité des cinq pays étudiés apparaît souvent avant le générique du programme, dans une sorte de prologue aux éditions hebdomadaires du ciné-journal. Dans les cas les plus emblématiques, les événements régionaux se confondent avec les fiches de présentation du programme, comme dans cette édition spéciale, datant du 12 août 1968, au sujet de la sortie du journal personnel de Che Guevara (fig. 1).

Figure 1. - Publication du journal personnel de Che Guevara (Noticiero, 12/09/1968).

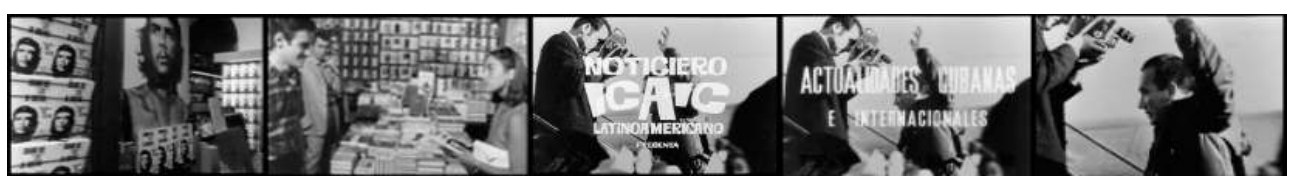

Source : Inamediapro

11 Ces premières observations indiquent l'existence d'une hiérarchie de l'information dans laquelle prévaut l'intérêt pour la couverture régionale. D’ailleurs, ce qui a motivé la première sortie du Noticiero sur le grand écran en 1960 est précisément la grande tournée du président Osvaldo Dorticos en Amérique latine. «Ce fut une première qui est rentrée dans l'Histoire ", explique Jerónimo Labrada, technicien du son chez Noticiero. C'était en effet la première et dernière visite d'un chef d'État cubain dans de nombreux pays latino-américains avant l'embargo américain et la censure des régimes militaires consolidés au cours des décennies 1960-1970.

\subsection{Les « années de plomb » militaires : nouvelles conditions de production de l'information}

12 L'analyse quantitative du corpus intégrant uniquement les actualités latinoaméricaines montre que la couverture des années avant les coups d'État militaires est beaucoup plus importante que celle d'après. Du point de vue narratologique, il y a un mouvement croissant de production qui s'intensifie au moment des coups d'État, avec la multiplication des émissions et une conséquente extension du récit journalistique. Ensuite, la couverture se réduit par la raréfaction des éditions produites pendant les régimes militaires, ce qui équivaut à une compression du «macro-récit » filmique (Arquembourg, 2005).

13 À titre d'illustration (fig. 2), et pour ne parler que des cas les plus emblématiques, nous constatons dans la couverture du Chili une certaine régularité entre 1960 et 1970 
(moyenne d'une émission par an) qui contraste avec l'intensité de la couverture dans l'imminence et le déroulement du coup d'État entre 1971 et 1973 (moyenne de quatre émissions par an). Nous arrivons à des chiffres similaires dans le cas du Brésil (16 émissions entre 1961 et $1965 ; 4$ émissions entre 1965 et 1978) et de l'Uruguay (9 émissions entre 1962 et $1972 ; 1$ émission en 1973).

Figure 2. - Cartographie historique du corpus : difficiles conditions de production après les coups d'État.

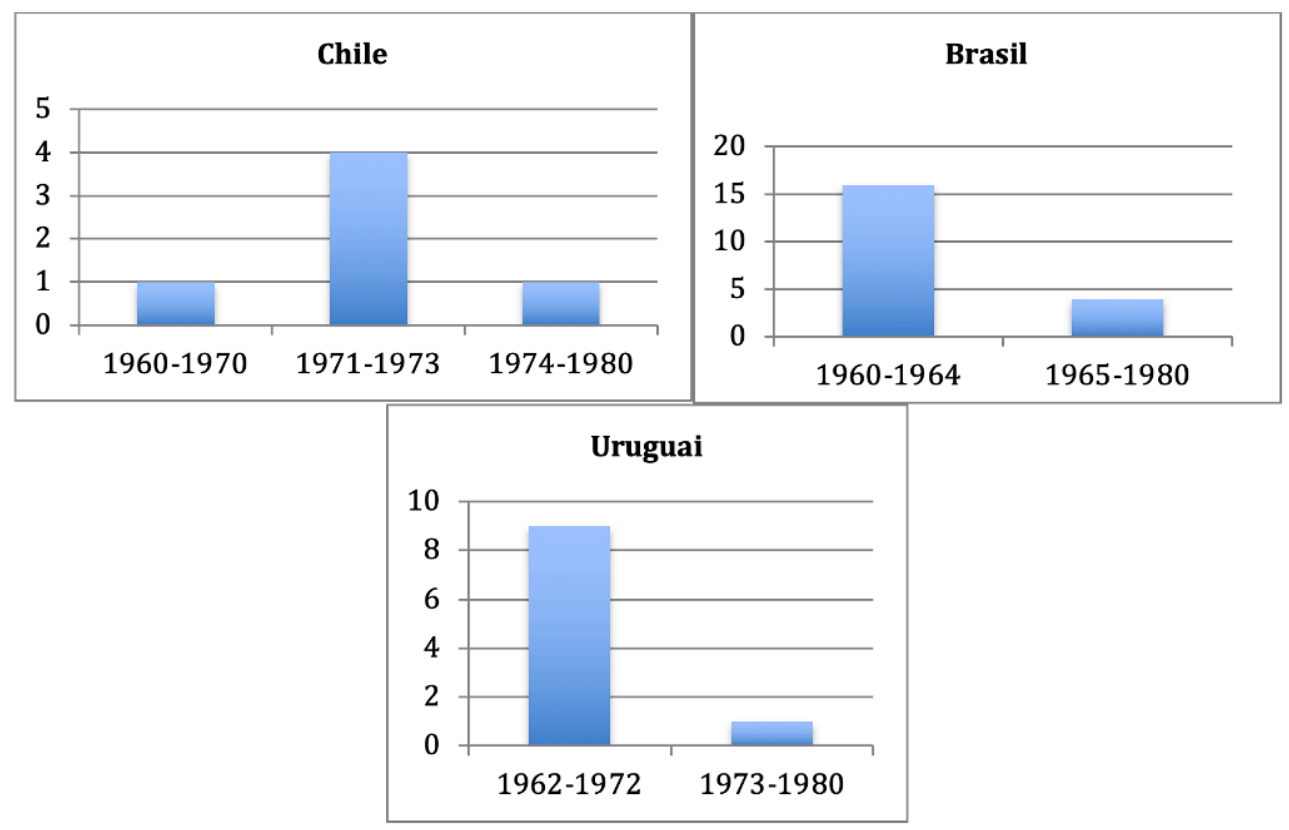

Ces constats nous permettent de conclure qu'à l'instar des actualités internationales se focalisant davantage sur les pays du Sud attaqués par les États-Unis, l'actualité latinoaméricaine est privilégiée dans le contexte des dirigeants populaires socialistes et/ou anti-impérialistes arrivés au pouvoir dans les années 1960 et 1970, se montrant solidaires de la révolution et de la souveraineté cubaines. En contraste, lorsque ces pays tombent sous l'emprise des régimes dictatoriaux, ils ne sont plus au centre de l'agenda du Noticiero. Durant les « années de plomb » d'Amérique latine, la production se limite à des émissions sporadiques - une fois tous les deux ou trois ans - qui font le bilan désastreux des régimes dictatoriaux militaires et de l'emprise nord-américaine sur la région.

Ces premiers éléments d'analyse narratologique quantitative valident le point de départ de cette étude, indiquant un important changement de paradigme dans la couverture de l'actualité latino-américaine avant et après les coups militaires. Lorsque nous analysons la matière filmique (discours, images, narration, bande sonore, photographie, cadrage, montage) qui configure le récit ciné-journalistique du Noticiero entre 1960 et 1990, nous observons une expérimentation croissante du langage à partir des années 1970 qui traduit la nouvelle donne géopolitique. Le blocus nord-américain de 1962 et la censure médiatique des régimes dictatoriaux latino-américains à partir des années 1970 renforcent l'isolement de Cuba. Le manque de source d'information et les entraves à la circulation d'images au niveau continental dessine alors de nouvelles conditions de production des actualités cinématographiques cubaines. 
Selon les cinéastes du Noticiero que nous avons pu interviewer, cette conjoncture géopolitique explique l'émergence de l'esthétique expérimentale qui est aujourd'hui considérée comme la marque de fabrique du Noticiero. "Nous avons toujours dû improviser avec ce que nous avions, c'est-à-dire : rien ", résume le photographe Julio E. Simoneau Martinez. À son tour, le réalisateur Manuel Pérez Paredes raconte qu'à l'époque, l'ICAIC avait seulement trois sources d'informations: "D'abord, le service Reuters, le seul auquel nous avons souscrit, puis les camarades cinéastes qui apportaient du matériel journalistique ou documentaire et, enfin, la légendaire antenne parabolique géante située à Guanabo, juste en face de Miami. »

Ces difficultés pratiques issues du contexte de la Guerre froide ont fait émerger un langage expérimental unique, fait de collages de divers matériaux visuels et sonores, de photomontages, de plans filmés sur la télévision et de mouvements de caméras sur les journaux et les magazines. Les entretiens réalisés nous permettent alors de ratifier une idée déjà présente dans le manifeste du cinéaste Julio García Espinosa, Por un cine imperfecto (1970). Les conditions de production de l'information journalistique en période d'embargo et de censure ont fonctionné comme un moteur d'expérimentation et de critique, marquant le point culminant de l'esthétique expérimentale et consolidant une forme d'art politique, faite d'improvisation et de créativité.

L'édition ci-dessous sur le Chili, du 5 juillet 1973 (fig.3), rend visible et lisible les conditions de production du Noticiero au moment du coup d'État militaire. Cette séquence illustre la première attaque contre le palais de la Moneda uniquement à partir de photos de journaux, de magazines et, curieusement, d'une carte du quartier présidentiel sous-titrée en anglais. La rareté des images en mouvement fonctionne ici comme un double signe. Signe « indiciel » (Peirce, 1998) révélateur de l'imminence du coup d'État qui allait entraîner deux mois plus tard la mort de Salvador Allende. Signe «symbolique » (Peirce, 1998) d'un langage expérimental qui doit contourner le faible accès aux sources d'information, en particulier celles provenant des anciens régimes latino-américains solidaires à Cuba.

Figure 3. - Un langage expérimental qui contourne la rareté de sources d'images (Noticiero, 05/07/1973).

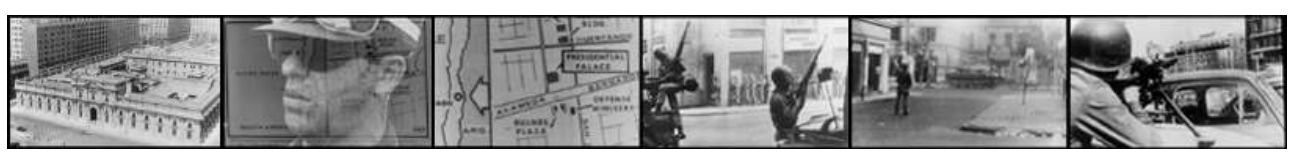

Source : Inamediapro

\subsection{Quand les coups d'État transforment le cadrage de l'actualité régionale}

19 Ces analyses s'affinent et se renforcent lorsque nous nous penchons sur les images et les discours qui configurent le récit filmique du Noticiero. L'étude du "cadrage » journalistique montre que les coups d'État militaires en Amérique latine constituent un tournant narratologique du récit d'actualité cinématographique cubain, sous forme d'une véritable rupture esthétique. Dans chacun des pays étudiés, la prise de pouvoir par les généraux marque le point narratif à partir duquel changent l'agencement 
temporel de l'intrigue, le rôle des personnages, les paradigmes discursifs et, enfin, la dimension expérimentale du langage visuel mobilisé.

Résumés dans le tableau ci-dessus (fig. 4), ces éléments d'analyse montrent que la transformation du récit cubain dans les années 1970 et 1980 consolide une esthétique expérimentale unique qui se caractérise par un langage hybride - situé entre le journalisme télévisé et le cinéma documentaire - et une lecture dialectique (visuelle et sonore) des actualités régionales projetées sur les écrans de cinéma.

Figure 4. - Caractérisation du corpus : avant et après les coups d'État.

\begin{tabular}{|c|c|c|}
\hline & Avant & Après \\
\hline Images & $\begin{array}{l}\text { - Cadrage: prédominance des } \\
\text { plans généraux/ d'ensemble/ } \\
\text { américains. } \\
\text { - Mouvement de caméra et } \\
\text { rythme du montage lents. }\end{array}$ & $\begin{array}{l}\text { - Photomontages et collages des images } \\
\text { d'actualité, d'archive ou abstraites. } \\
\text { - Beaucoup de zoom et gros plans (affection) } \\
\text { - Rythme du montage accéléré et inconstant. } \\
\text { Résonance des sujets suggère des points de vue } \\
\text { énonciatifs et cadres normatifs (jugement). }\end{array}$ \\
\hline Discours & $\begin{array}{l}\text { - Narration avec point de vue } \\
\text { omniscient (focalisation zéro). } \\
\text { - Système de personnages : } \\
\text { « dirigeants + peuples " (alliés } \\
\text { politiques/idéologiques) X X } \\
\text { « américains + comparses ». }\end{array}$ & $\begin{array}{l}\text { - Forte responsabilité discursive. Jeux de } \\
\text { langage fonctionnent comme des recours } \\
\text { énonciatifs. } \\
\text { - Système de personnages : « dirigeants latinos } \\
\text { (soumis à l'intrigue) x peuple (dynamique, } \\
\text { capable de changer l'intrigue)» }\end{array}$ \\
\hline Musique & $\begin{array}{l}\text { - Chansons populaires } \\
\text { folkloriques. } \\
\text { - Bande sonore très propre. }\end{array}$ & $\begin{array}{l}\text { - Chansons de protestation. } \\
\text { - Discours, musiques et bruits intercalés. }\end{array}$ \\
\hline Conclusion & $\begin{array}{l}\text { Esthétique visuelle et sonore } \\
\text { classique construit des portraits } \\
\text { idylliques des pays étudiés. }\end{array}$ & $\begin{array}{c}\text { Langage expérimental - fait des images } \\
\text { cinématographiques, documentaires et } \\
\text { fictionnelles - construit des portraits critiques } \\
\text { et éloignés des pays étudiés. }\end{array}$ \\
\hline
\end{tabular}

21 Dans l'analyse de la représentation des pays latino-américains avant les coups d'État, nous observons que les gouvernements populaires au pouvoir - davantage incarnés par les figures du président chilien Salvador Allende et les dirigeants brésiliens Joao Goulart et Leonel Brizola - sont présentés comme des "amis » ou des «frères » de Cuba, solidaires de la cause révolutionnaire et anti-impérialiste. Le récit filmique cubain soulève souvent l'appui et la satisfaction des peuples latino-américains face aux avancées de tels gouvernements (nationalisation des entreprises américaines, redistribution des richesses, réforme agraire, alphabétisation, etc.). Ce " point de vue " (Aumont, 1983) cubain construit un système d'actants qui oppose deux personnages collectifs : d'un côté, la figure unificatrice des gouvernants et leurs peuples et, de l'autre côté, la figure unique de l'ennemi américain. La construction narrative de ce "système de personnages » (Lits, 2008) s'appuie sur un champ discursif binaire, composé de qualificatifs péjoratifs, opposant les acteurs " socialistes » aux « fascistes ", les situations de "souveraineté nationale » à la "soumission nord-américaine » et, enfin, le « communisme » au « capitalisme ».

Au niveau des images qui matérialisent la «fable» $(E c o, 1985)$ de la période avant les coups d'État, nous retrouvons une esthétique journalistique classique, tant en ce qui concerne le cadrage photographique (plans généraux et américains), que le mouvement de la caméra et le rythme de montage (tous deux lents). Au niveau de la musique, nous constatons la prégnance des chansons populaires nationales de type folklorique et 
spécifiques à chacun de ces pays. Nous constatons ainsi que dans la représentation latino-américaine d'avant la période militaire, l'esthétique visuelle et sonore du Noticiero soutient la construction de portraits très positifs, voire idylliques, des pays voisins, en mettant en évidence leurs richesses naturelles, culturelles, économiques et industrielles.

En revanche, dans la période après les coups d'État militaires, ces portraits changent radicalement. À partir de ce point, les émissions rendent intelligible le fossé qui sépare les nouveaux régimes - désignés comme des "fascistes", des "assassins", des "gorilles» au service des "yankees »- et leur peuple qui subit la répression. Ici, le système de personnages est construit par une opposition entre la classe gouvernante et le peuple. Cette grille de lecture dialectique construit des portraits opposés au sein de chacun des pays étudiés : d'un côté, la représentation de la classe dirigeante bourgeoise qui exploite des richesses nationales (pétrole, mines, sources aquifères, etc.) et, de l'autre côté, un portrait sombre du peuple illettré, en état de famine ou de dénutrition.

$\mathrm{Au}$ niveau des images, nous constatons que l'esthétique visuelle gagne en connotation dramatique, soit par le remplacement des plans d'ensemble par des gros plans, soit par le ralentissement des plans montrant des scènes de violence (massacres, tortures, humiliation), soit par l'accélération du montage et l'usage des images fictionnelles, conceptuelles et abstraites dans la couverture des actualités régionales. En ce qui concerne le travail de montage, il y a une récurrence de métaphores et de superpositions d'images qui produisent un effet de contraste visuel chargé en argumentation.

25 Au niveau du son, celui-ci accompagne l'intensification du récit, avec des collages de musiques, discours et bruits divers (son de tirs de pistolet, de canon, de bombe, explosion, etc.). Les chansons de protestation deviennent particulièrement importantes et font également l'objet d'un montage sonore dialectique. D'une part, ces chansons accompagnent des scènes d'émeutes et de manifestations populaires. De l'autre, des sons de percussions et tambours, ainsi que des extraits musicaux du groupe d'expérimentation sonore de l'ICAIC accompagnent les images de confrontation et de violence entre la population et les forces de l'ordre. Concernant l'instance de narration, on note la prédominance d'un point de vue omniscient et d'une modalité discursive de forte responsabilité énonciative (List, 2008).

26 Ces éléments d'analyse montrent que, dans la couverture des actualités latinoaméricaines dans la période d'après les coups d'État, la compression du récit filmique analysé ci-dessus en termes quantitatifs correspond ici à un changement qualitatif de consolidation de l'expérimentation esthétique dans la représentation des Amériques. Depuis les coups d'État, la rareté des sources d'information journalistique conduit les cinéastes de l'ICAIC à investir plus que jamais dans l'expérimentation et la création artistiques. En mettant en perspective des sources cinématographiques, journalistiques et fictionnelles, le langage expérimental du Noticiero qui émerge dans les années 1960-1970 se situe à mi-chemin du télé-journalisme, du cinéma documentaire et de l'art révolutionnaire. C'est ainsi que prend forme le langage audiovisuel expérimental qui deviendrait la principale marque esthétique et politique du Noticiero (Del Valle Dávila, 2015).

27 Les analyses développées jusqu'ici nous permettent alors de répondre aux deux premières questions posées dans cet article. L'étude quantitative et qualitative du corpus rend intelligible la manière dont l'agenda, le cadrage et le récit des actualités 
américaines changent en fonction des conditions de production-circulation des images dans un continent stratégique dans la géopolitique de la Guerre froide. Si la représentation péjorative des États-Unis se maintient pendant trenteans, la couverture des actualités latino-américaines bascule au rythme des coups d'État militaires régionaux. Après avoir défini les principales marques (discursives, visuelles, sonores) du langage expérimental du Noticiero, il convient maintenant de présenter les enjeux sémiotiques de cette expérimentation afin de mieux saisir le rôle joué par les images (et la voix off) dans la construction des représentations des Amériques pendant les « années de plomb» du continent.

\section{Le rôle des images dans la représentation des Amériques : enjeux sémiotiques du langage expérimental}

28 Afin de saisir le rôle joué par les images dans la couverture des actualités américaines du Noticiero, nous développerons ici une analyse plus fine des procédés sémiotiques (visées, valeurs, significations) qui traversent la production et l'usage, par les cinéastes de l'ICAIC, des images fixes, des images en mouvement et des signes discursifs dans la représentation des Amériques. Pour traiter de manière représentative (et non exhaustive) le grand répertoire d'images qui configurent les actualités américaines dans le corpus étudié, nous avons créé des catégories d'analyse renvoyant aux différentes visées sémiotiques - rhétorique, énonciative et performative - de ces images et des commentaires qui les accompagnent. Dans cette partie, nous répondrons alors à la dernière dimension de notre problématique. Il s'agira d'analyser la manière dont les images des Amériques véhiculées par le Noticiero répondent à des stratégies propagandistes et révolutionnaires faisant des actualités continentales un enjeu de luttes idéologiques autour de l'écriture de la mémoire et de l'histoire de l'Amérique latine.

\subsection{La rhétorique du montage : didactisme, idéologie et satire}

Pour penser ce qui serait la "rhétorique visuelle» (Dondero, 2013) du Noticiero, analysons tout d'abord la dimension didactique des images qui configurent les représentations des Amériques. Il s'agit de la seule catégorie régulièrement trouvée dans le sous-corpus de la période avant les coups d'État. Utilisée notamment dans la couverture des actualités régionales, cette visée didactique est matérialisée par un dispositif visuel consistant à associer des images documentaires et animées (plans, cartes, photos aériennes ou de paysages). Ce langage expérimental a pour but d'(in)former le public cubain sur l'emplacement géographique et les richesses naturelles des pays latino-américains, alors cibles d'attaques nord-américaines dans une forme de Guerre froide continentale.

Nous trouvons des exemples emblématiques de ce dispositif expérimental visuel dans ces deux séquences d'images. La première, du 5 juillet 1967, illustre une attaque nordaméricaine contre la Bolivie (fig. 5). La seconde, du 28 juillet 1979, trace une cartographie des invasions nord-américaines dans le monde (bases militaires et soldats dans plus de 50 pays) au sein d'une édition sur le Chili d'Augusto Pinochet (fig. 6). 
Figure 5. - Illustration didactique de l'attaque états-unien en Bolivie (Noticiero, 05/07/1967).

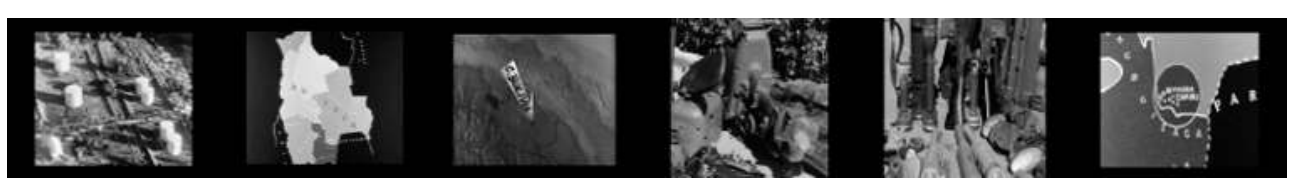

Source : Inamediapro

Figure 6. - Cartographie didactique des invasions nord-américaines à l'étranger (Noticiero, 28/07/1979).

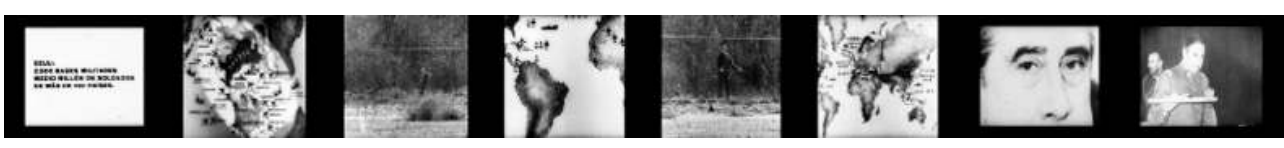

Source : Inamediapro

31 L'expérimentation du langage matérialisée ici par le montage d'images documentaires et animées rend visibles les difficiles conditions de production décrites plus haut. L'absence d'image satellite ou du terrain de guerre, amène les cinéastes cubains à improviser avec d'autres sources de production de sens : les tables d'animation et le montage rhétorique. Le langage expérimental qui en résulte est d'autant plus fort que le récit visuel ainsi configuré oscille entre le didactisme et la rhétorique. Celle-ci est notable dans la deuxième séquence qui présente Pinochet comme l'icône de l'asservissement (figure du majordome) des leaders latino-américains au projet impérialiste des États-Unis. D’une séquence à l'autre, nous observons la progression du langage expérimental didactique du Noticiero qui entre 1960 et 1980 assume des contours argumentatifs plus explicites.

La rhétorique du montage présente aussi une visée idéologique lorsqu'elle joue sur l'association des idées (iconiques et discursives) afin de cristalliser des positionnements politiques. Aux images produites en table d'animation, s'ajoute l'usage récurrent des signes discursifs qui intègrent la matière audiovisuelle du Noticiero sous une visée critique ou propagandiste, tel que l'illustrent respectivement les séquences sur le Chili du 7 février 1982 (fig. 7) et sur l'Uruguay du 28 juillet 1961 (fig. 8).

Figure 7. - Le Chili d'Augusto Pinochet (Noticiero, 07/02/1982).

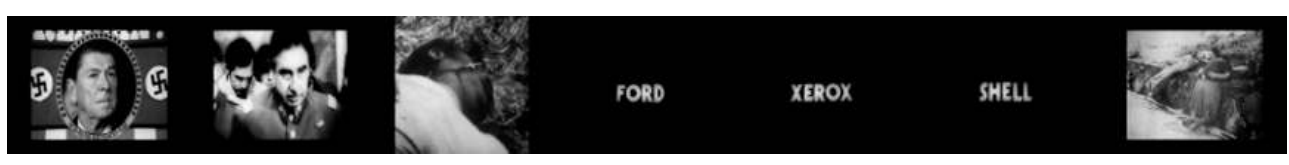

Source : Inamediapro

Figure 8. - Le peuple uruguayen avec Cuba (Noticiero, 28/07/1961).

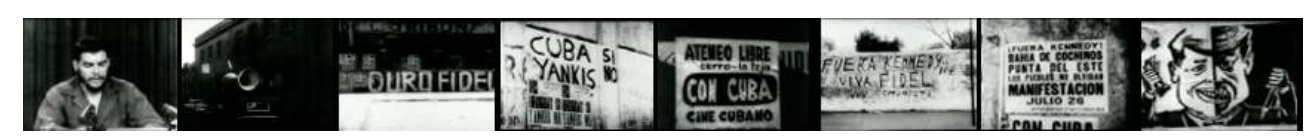

Source : Inamediapro

33 La critique idéologique cubaine se fait fortement sentir dans la première séquence d'une édition faisant le bilan des six années de gouvernement du général Augusto 
Pinochet au Chili. Dans ce passage, nous observons comment le montage rhétorique joue sur l'association de signes iconiques (l'emblème nazi), discursifs (marques d'entreprises américaines) et documentaires (corps de victimes) pour signifier le capitalisme sous le signe du fascisme meurtrier, dans une représentation des Amériques marquée par la Guerre froide.

La seconde séquence d'images est issue d'une édition couvrant la rencontre de l'Organisation des États américains (OEA) en Uruguay. Elle illustre les longs plansséquences sur des affiches de rue que nous trouvons régulièrement dans les corpus. Notons que, lorsque ce récit visuel intègre les signes discursifs des affiches en soutien à Fidel Castro et Cuba, il confère une signification, c'est-à-dire un "dire ", à ce qui est «dit» (Ducrot, 1985) par les énoncés des affiches. Ce «dire» se trouve alors exprimé par la voix du narrateur: "Comme toute l'Amérique latine, le peuple d'Uruguay exprime sa solidarité à notre révolution socialiste. Dégage Kennedy, c'est le cri unanime du peuple latino-américain contre les monopoles. " La rhétorique du montage ainsi construit permet de placer le peuple uruguayen dans la lutte internationaliste (sous la figure de Che Guevara) pour la souveraineté cubaine et contre l'impérialisme "yanqui », alors que la classe dirigeante nationale passe progressivement du côté nord-américain à chacune des rencontres diplomatiques dans le cadre de l'Organisation des États américains (OEA), à Punta del Este, Uruguay.

Hors de l'écran, l'avancée des dictatures latino-américaines consolide les plans de "l'opération Condor" et l'importante couverture des réunions de l'OEA faite entre 1962 et 1967 fonctionne comme la grande scène de la critique satirique du Noticiero - la troisième visée du montage rhétorique analysée ici. Dans la couverture périodique de l'événement (qualifié de «mascarade impérialiste contre la révolution cubaine»), nous avons identifié un paradigme argumentatif régulier consistant à utiliser l'humour satirique pour minimiser la politique, la posture, la parole et, même, la tenue vestimentaire des dirigeants américains et leurs "comparses" latinoaméricains. La critique satirique s'appuie ici sur des figures animales (lions marins, rats, dauphins, singes) pour inférioriser l'ennemi américain.

Dans la première séquence datant du 5 février 1962 (fig. 9), le montage alterné entre les représentants politiques à l'OEA et des otaries sur la plage amène progressivement les cris des otaries à se substituer aux discours des délégués politiques.

Figure 9. - Réunion de l'OEA à Punta del Este (Noticiero, 05/02/1962).

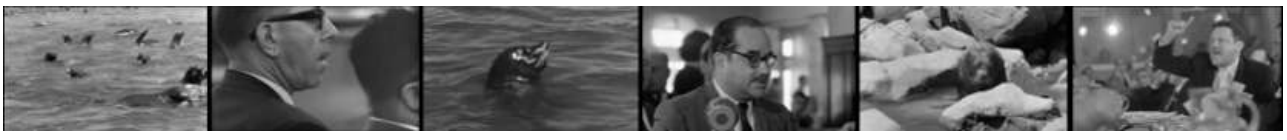

Source : Inamediapro

37 Neuf mois plus tard, le 12 novembre 1962 (fig. 10), le sujet est à nouveau à l'ordre du jour et, cette fois-ci, les conseillers nord-américains et leurs alliés régionaux sont comparés à des rats. Dans l'ouverture de l'édition, le narrateur annonce «Attention, attention, ceci est un appel fédéral du Pentagone aux serviteurs de l'OEA. À l'ordre du jour: sujet Caribe ", tandis qu'une séquence d'images filmés suit le parcours des rats qui sortent des égouts, traversent les rues et, enfin, s'entassent les uns sur les autres dans un tout petit espace. La métaphore représente ici la trajectoire des dirigeants américains vers une des rencontres de l'OEA. 
Figure 10. - Appel d'urgence aux membres de l'OEA (Noticiero, 12/11/1962).

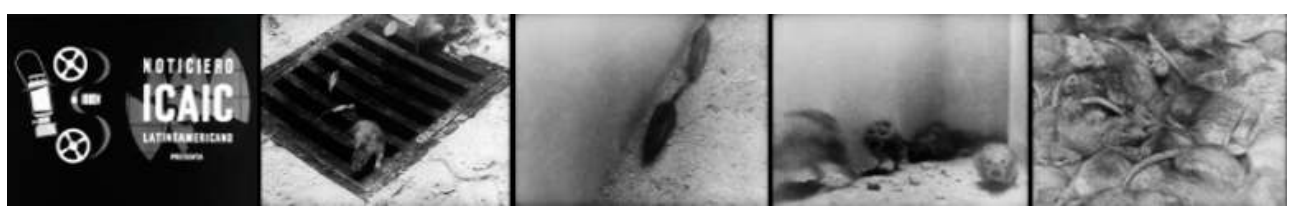

Source : Inamediapro

États-Unis dans le Noticiero reproduit une rhétorique classique des propagandes idéologiques dans la scène médiatique de la Guerre froide. Plus particulièrement, dans les actualités cubaines, la représentation animalisée de l'ennemi "impérialiste » cherche moins à le déshumaniser qu'à l'inférioriser par l'humour satirique. Produit du montage rhétorique, la satire ciné-journalistique du Noticiero cherche ainsi à renverser les représentations - et l'ordre géopolitique - dominantes dans le continent américain.

Figure 11. - Le portrait de Richard Nixon dans la couverture de la campagne électorale américaine de 1972 (Noticiero, 03/11/1972).

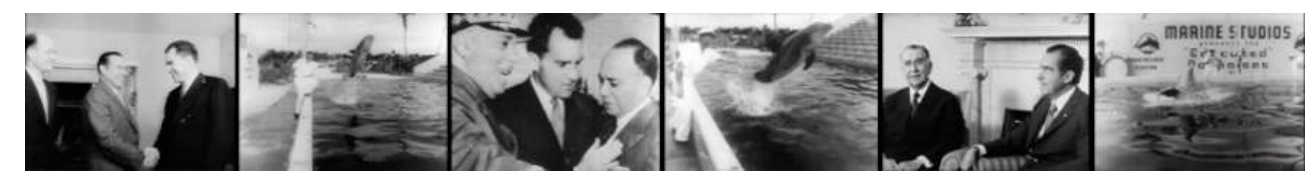

Source : Inamediapro

\subsection{L'énonciation visuelle du montage dialectique}

Dans la couverture des «années de plomb » de l'Amérique latine, les réalisateurs du Noticiero cherchent à faire comprendre au public cubain que la Guerre froide continentale a lieu à l'intérieur de chacun des pays latino-américains. Pour signifier la fracture sociale opposant le peuple à la bourgeoise, ils utilisent un montage dialectique, propre au cinéma soviétique des années 1920, consistant à évoquer des parties contradictoires de la réalité sociale afin de transformer la perception de celle-ci. Inspirés par les mouvements du réalisme socialiste russe et du néoréalisme italien, les producteurs du Noticiero multiplient les gros plans focalisés sur les visages pour renforcer la charge dramatique qui rend visible la souffrance des peuples latinoaméricains. 
Comme l'illustre ce reportage sur la situation post-coup d'État en Bolivie, datant du 14 février 1974 (fig. 12), le montage dialectique des images en mouvement construit une critique idéologique dont la signification est renforcée par le commentaire du narrateur: "Tandis que les banques profitent des réserves nationales minières, les gens travaillent pour payer un coût de la vie qui ne cesse pas de grandir.» L'exploitation et la souffrance du peuple bolivien sont ici exprimées par les visages d'indigènes qui incarnent les victimes du « néo-colonialisme » nord-américain.

Figure 12. - Portrait de la fracture sociale en Bolivie (Noticiero, 14/02/1974).

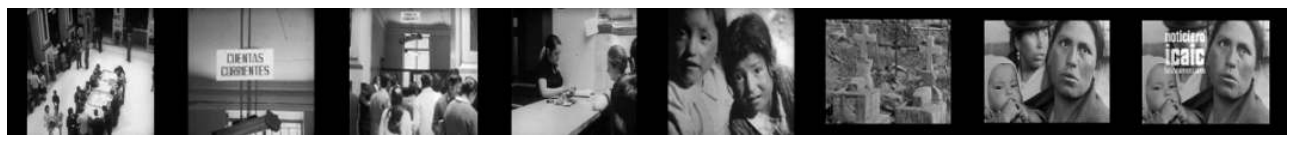

Source : Inamediapro

Cette séquence illustre l'usage régulier des gros plans qui, remplaçant les plans généraux, caractérisent la couverture des actualités dans la période post-coups d'État. Ce procédé permet aux cinéastes cubains de mettre l'accent sur certains visages emblématiques des populations nationales: peuples autochtones, habitants des bidonvilles, des périphéries et sans-abri. Hérité de l'esthétique cinématographique russe, ce type de cadrage photographique suscite une sorte d'investissement sensible de la part des spectateurs, alors très proche de la catégorie d' "image-affection " décrite par Gilles Deleuze (1983) comme une surface de signification et d'identification sociale, politique et idéologique pouvant amener à l'action contestée.

Tout aussi représentative du montage dialectique qui prend forme dans la période postcoup d'État, cette séquence sur le Brésil, du 7 mars 1978 (fig. 13), dessine deux portraits nationaux en confrontation. Ce n'est pas l'instance narrative qui oriente la réception dans une visée critique, mais le montage des images fixes (photographies et cartes postales) et la bande sonore qui permettent de confronter les portraits nationaux. La première séquence d'images reproduite ci-dessous émerge sur fond de bossa nova. Pendant que les photomontages défilent, le narrateur se limite à dire une seule phrase : «Brésil, 1978. Ceci est l'un des visages du miracle brésilien. » La séquence dure deux minutes et la narration ne fait qu'introduire et conclure l'énonciation par une critique idéologique qui dépeint un sombre portrait des dictatures militaires. Quand nous passons à la deuxième séquence d'images, la bande sonore constituée d'une samba en crescendo renforce la charge dramatique de l'énonciation du narrateur: "Brésil, 1978. Celle-ci, c'est l'autre face du miracle économique brésilien. Son vrai visage. L'impérialisme yanqui essaie de montrer au monde une image sophistiquée du Brésil actuel en échange du rôle de police que ce pays joue dans l'hémisphère. »

Figure 13. - Les deux faces du « miracle économique » au Brésil (Noticiero, 07/03/1978).

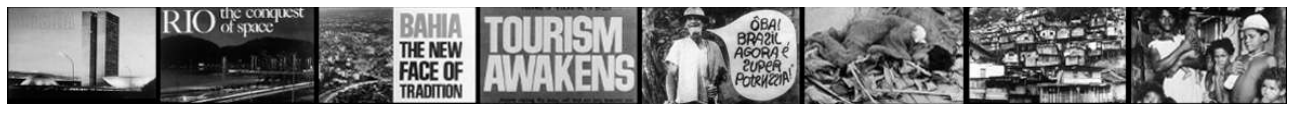

Source : Inamediapro

Ces deux dernières séquences en Bolivie et au Brésil rendent intelligible une dialectique visuelle et sonore, récurrente dans le corpus, qui fonctionne comme un agencement sémiotique central de l'énonciation visuelle post-coup d'État. L'effet de 
" distanciation " généré par la juxtaposition contrastée des images et des musiques vise à informer et conscientiser le spectateur sur l'existence d'une lutte de classe qui déchire chacun des pays sous l'emprise des dictatures militaires. L'« énonciation visuelle " (Dondero, 2013) créée par ce type de montage dialectique invite le spectateur à "prendre ses distances " avec la réalité pour mieux la voir, politiquement et poétiquement.

\subsection{La performativité des images à travers le métalangage}

l'analyse de la représentation dialectique de l'actualité latino-américaine, nous observons que l'énonciation visuelle du Noticiero porte une visée pragmatique qui cherche, non seulement à signifier la réalité socio-politique régionale, mais aussi et surtout à agir sur celle-ci dans le présent. Nous touchons ici à la question de la performativité du langage expérimental du Noticiero qui a pris forme à partir des années 1970.

Comment alors penser la dimension pragmatique - c'est-à-dire la capacité d'action et d'influence - des images qui configurent la double représentation des Amériques dans le Noticiero? En s'appuyant sur la théorie pragmatique des actes langagiers de John Austin et la sémiotique de Charles S. Peirce, la sémioticienne Jocelyne Arquembourg nous indique une méthode d'analyse pertinente pour l'étude de la performativité visuelle du Noticiero par cette question : «comment l'acte de montrer des images est-il investi de valeurs particulières par les interactants » $(2010: 173)$ ?

47 En effet, nous observons qu'à chaque fois que le narrateur du Noticiero s'engage à "révéler» ou à "rendre publique " l'histoire non officielle des dictatures latinoaméricaines, dans un contexte de forte censure médiatique, il accomplit un acte de langage performatif. Le véhicule de cette action est le discours métalinguistique - le commentaire sur l'image - fréquemment mobilisé par l'instance de narration qui assume une forte responsabilité énonciative et garantit le contrat de communication avec le spectateur. Voici quelques exemples de cette ressource linguistique que l'on retrouve régulièrement dans le corpus étudié: "Ces soldats recrutés reçoivent la bénédiction. Mais plus tard, ils recevront des coups de feu »; «Écoutez la voix du médecin...» (Noticiero, 5 juin 1967) ; «Regardez ce balcon, quelque chose va se passer (Noticiero, 24 avril 1967) »; "En Rhodésie, ces images rappelant les camps de concentration nazis ont été prises par une agence de presse occidentale capitaliste " (Noticiero, 16 juillet 1976).

Dans ce type de "révélation » médiatique (Arquembourg, 2010), on constate que le narrateur ne se limite pas au caractère « indiciel » de l'image qui, sous le mode de la constatation, affirme l'existence du fait/événement, comme le suggère Roland Barthes (1980) dans la théorie du « ça a été ». Le narrateur va plus loin, en justifiant l'importance et la valeur historique d'un telle "révélation » au sujet des " horreurs » des dictatures militaires qui, avant la révélation du Noticiero, n'avaient pas d'existence publique. Une telle qualification (valeur) de l'acte de la « révélation » fonctionne donc comme un acte de langage performatif qui cherche à agir non seulement sur le sens de ce qui est dit et montré sur l'écran (acte illocutoire), mais qui cherche aussi à agir sur la réalité extra-filmique (acte perlocutoire) (Austin, 1970), c'est-à-dire à encourager le spectateur au combat anti-impérialiste et à l'action révolutionnaire. 
49 La séquence ci-dessous, datant du 20 septembre 1973, sur le Chili et son "armée meurtrière " (fig. 14), illustre de manière emblématique la dimension pragmatique de la ressource métalinguistique souvent utilisée par le narrateur du Noticiero. Dans ce passage, lorsque le narrateur décrit le contenu et le moment de l'enregistrement des images, il qualifie leur valeur historique et nous met au défi d'agir, en disant :

Ces images ont été filmées lors de la première tentative fasciste en juin 1973. Le caméraman a continué à filmer tandis que les fascistes lui tiraient dessus, le regardant de sang-froid. Ces images anticipent les massacres qui seront déclenchés par les fascistes des mois plus tard. Frère chilien, frère latino-américain, n'oubliez pas ces visages, ce sont vos tueurs. (Noticiero, 20 septembre 1973)

50 Comme nous pouvons le voir, l'utilisation du métalangage permet non seulement de re-signifier les images selon le «point de vue » cubain, mais surtout de les interpréter en fonction du contexte de production et de diffusion. Comme le souligne Marc Ferro à cet égard :

Le film contribue à construire une contre-histoire officieuse, en partie libérée des archives écrites qui ne sont rien d'autre que la mémoire conservée par nos institutions. Jouant un rôle actif en contrepoint de l'histoire officielle, le film devient un agent de l'histoire en tant qu'il contribue à une prise de conscience. (1993 : 13)

51 Cette narration qui "révèle » le sens de l'Histoire fonctionne comme la principale ressource performative du langage expérimental du Noticiero. Les actualités cubaines cherchent ainsi à agir sur le spectateur et à le conduire à l'action : descendre dans la rue, manifester, résister, s'armer et combattre. À ce propos, Santiago Álvarez parlait en termes de "guerre contre l'accommodement, l'inertie, le conformisme et l'insuffisance », en décrivant l'importance de s'engager dans la réalité : « Ainsi, on perd la peur des mots chargés en contenu péjoratif [...]. Nous devons sauver des concepts et des positions concernant la réalité et l'art qui ont subi des déformations bureaucratiques. La peur de tomber dans l'apologétique [...] est une peur irréelle et pernicieuse. » $(2012: 15,57)$

Figure 14. - Commentaire sur l'image de l'armée chilienne (Noticiero, 20/09/1973).

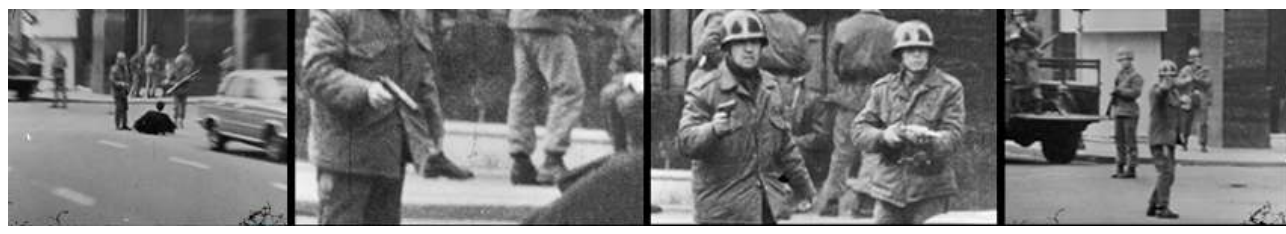

Source : Inamediapro

52 Nous trouvons un cas illustratif de ce type de récriture performative du sens de l'Histoire dans l'édition-hommage consacré au décès du leader chilien Salvador Allende juste après la prise du palais de la Moneda (fig. 15). Sur l'écran, la mort de Salvador Allende le 11 septembre 1973 apparaît comme le signe de la fin d'une époque, mais non pas de la fin de l'Histoire selon le « point de vue » cubain. Dans l'édition-hommage que le Noticiero lui a consacrée et notamment dans la séquence finale ci-dessous, la performativité est fortement ressentie. Dans la première image, Allende déclare :

Je vous le dis avec calme, avec une totale tranquillité, je n'ai pas vocation d'apôtre ni de messie. Je n'ai pas l'âme d'un martyr ; je suis un lutteur social qui remplit une tâche que lui a confié le peuple. Mais que ceux qui veulent arrêter l'Histoire et ignorer la volonté majoritaire du Chili l'entendent : sans avoir l'âme d'un martyr, je 
ne ferais pas un pas en arrière. Qu'ils le sachent, qu'ils l'entendent, qu'on le leur grave profondément: je quitterais La Moneda quand mon mandat sera terminé. Je défendrais cette révolution chilienne et je défendrais le gouvernement. Je n'ai pas d'autre choix. Il faudra me cribler de balles pour m'empêcher de réaliser le programme du peuple. (Noticiero, 20 septembre 1973)

Nous passons ensuite à la deuxième, la troisième et la quatrième image de cette séquence, en entendant la conclusion du discours d'Allende : «Et si on m'assassine, le peuple suivra sa route. Demain, d'autres compagnons vous rejoindront. Et le peuple suivra la révolution chilienne. » L'édition-hommage se termine par des coups de feu sur l'image d'un tireur d'élite - Salvador Allende lui-même - qui devient progressivement floue jusqu'à disparaître complétement. Allende s'est suicidé lors de la prise de contrôle de Moneda le 9 novembre 1973 mais, sur les écrans de cinéma cubains, il était toujours vivant, en image et en discours, car la lutte devait se poursuivre et le spectateur en faisait partie.

Figure 15. - Édition-hommage à la vie Salvador Allende, lors de sa mort (Noticiero, 20/09/1973).

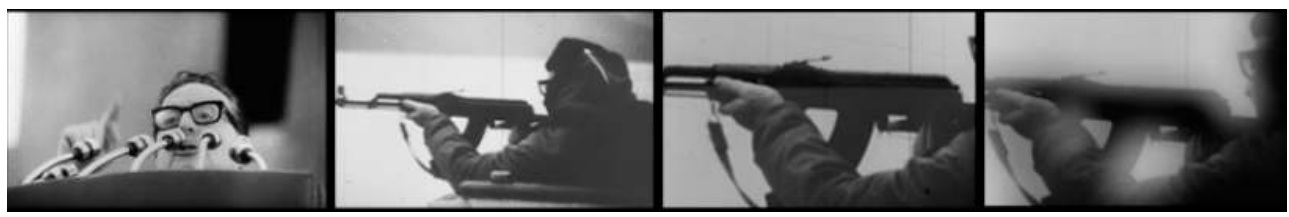

Source : Inamediapro

\section{Conclusion}

L'étude des actualités nord et sud-américaines gravées dans les pellicules du Noticiero ICAIC (1960-1990) met au jour le rôle des images dans la construction d'un certain point de vue cubain sur l'histoire d'une Guerre froide continentale. Il ressort de ce corpus d'archives audiovisuelles une représentation des Amériques déchirées entre le Nord et le Sud et divisée à l'intérieur des pays latino-américains.

Les entretiens avec les producteurs du Noticiero mettent en évidence les difficiles conditions de production de l'information dans un contexte de censure médiatique et d'isolement de l'île. Le langage expérimental qui en émerge place alors l'image cinéjournalistique au centre des luttes symboliques portant sur l'écriture d'une mémoire continentale. D'une part, la Guerre froide détermine l'agenda et le cadrage du Noticiero et, d'autre part, les coups d'État latino-américains transforment son récit cinéjournalistique.

56 L'analyse sémiotique des images (fixes et en mouvement) du corpus selon leurs propriétés et visées permet d'ébaucher une typologie renvoyant à leurs différents rôles. En tant que dispositifs visuels rhétoriques et énonciatifs, les images des Amériques véhiculées par le Noticiero auraient pour rôle de transformer la vision dominante de l'ordre social. En tant que dispositifs performatifs, les commentaires qui accompagnent ces images appellent les spectateurs à une mobilisation susceptible de renverser les représentations et l'ordre social dominants. Les images des Amériques ainsi analysées peuvent alors être comprises comme des dispositifs sémiotiques de l'action politique et artistique, propagandiste et révolutionnaire. 


\section{BIBLIOGRAPHIE}

Amiot-Guillouet Julie (2008), « Discurso oficial y mito del 'punto cero': una história en/de Cine Cubano ", N. Berthier (dir.), Cine y Revolución cubana: luces y sombras, Archivos de la Filmoteca, 59, 37-51.

ARAY Edmundo (1983), Santiago Álvarez: cronista de tercer mundo, Caracas : Cinemateca Nac.

ARQUEMBourG Jocelyne (2005), « Comment les récits d'information arrivent-ils à leurs fins? », Réseaux, 132(4), 27-50.

ARQUEMBourg Jocelyne (2010), « Des images en action. Performativité et espace public », Réseaux, 163(5), 163-187.

Aumont Jacques (1983), « Le point de vue. Énonciation et cinéma », Communications, 38, 3-29.

Austin John (1970), Quand dire, c'est faire, Paris : Seuil.

BARTHES Roland (1980), La chambre claire. Note sur la photographie, Paris : Gallimard.

Deleuze Gilles (1983), L'image-mouvement, Paris : Les Éditions de Minuit.

Del VAlle DÁVILA Ignacio (2015), Le nouveau cinéma latino-américain (1960-1974), Rennes : Presses universitaires de Rennes.

DíAz Mayra (2012), El Noticiero ICAIC y sus vocês, La Havane : Ediciones La Memoria, Centro Cultural Pablo de la Torriente Brau.

DONDERo Maria Giulia (2013), « Rhétorique visuelle et énonciation », Visible, 10, 9-31.

Ducrot Oswald (1985), Le dit et le dire, Paris : Les Éditions de Minuit.

Eco Umberto (1985), Lector in fabula, Paris : Grasset.

ENTMAN Robert M. (1993), Framing: Toward Clarification of a Fractured Paradigm, Journal of Communication, 43(4), 51-58.

FERRo Marc (1993), Cinéma et Histoire, Paris : Gallimard, Folio.

GARCíA EsPINOSA Julio (1970), Por un cine imperfecto, Caracas : Rocinante.

LiTs Marc (2008), Du récit au récit médiatique, Bruxelles : De Boeck.

McCombs Maxwell \& SHAw Donald L. (1972), « The Agenda-Setting Function of Mass Media », Public Opinion Quarterly, 36(2), 176-187.

PeirCe SANDers Charles (1998), The Essential Peirce: Selected Philosophical Writings, Volume 2 (18931913), Indiana University Press : Peirce Edition Project.

\section{NOTES}

1. Cette proposition est issue d'une recherche postdoctorale développée dans le cadre d'une bourse d'étude de l'INAthèque obtenu en 2017-2018.

2. Le choix de ces études de cas se justifie pour deux raisons : (1) l'importance quantitative de la couverture de ces pays, présentés par le Noticiero comme les plus répressifs et autoritaires de 
l'époque ; 2) le fait que les régimes militaires des pays mentionnés commencent et se terminent dans la borne temporelle qui délimite la recherche (1960-1990).

3. Raul Pérez Ureta, Julio E. Simoneau Martinez, Manuel Perez Paredes, Rebeca Chávez, Jorge Luis Sanchez, Raúl Rodriguez, Daniel Diez Castrillo, Jerónimo Labrada Hernández.

4. Issu de la théorie de l'« agenda-setting », le concept traduit le processus par lequel la couverture régulière d'un même sujet suggère au public «ce à quoi » il faut penser, selon une certaine hiérarchie de l'information.

5. Issu de la sociologie et de la psychologie, le concept de «cadrage " journalistique renvoie à la mise en avant de certains aspects d'un événement afin d'en promouvoir une certaine interprétation.

6. Les années 1960 marquent le processus de «soviétisation» de Cuba à partir de trois événements phares - l'invasion de la baie des Cochons (1961), la guerre des missiles (1962) et le blocus nord-américain (1962) - qui conduisent progressivement à l'alignement de l'île au régime soviétique.

7. Le rôle central de Cuba dans la promotion de la solidarité entre les peuples d'Asie, d'Afrique et d'Amérique latine tient non seulement à la première Conférence tricontinentale qui s'est tenue à La Havane en 1966, mais également au soutien de troupes révolutionnaires cubaines sur des champs de bataille au sein de ces trois continents.

\section{RÉSUMÉS}

Cet article développe une étude sémiotique des représentations des Amériques au sein du Noticiero ICAIC, des actualités cinématographiques produites et diffusées à Cuba entre 1960 et 1990. Au regard des conditions de production de l'information dans le contexte de la Guerre froide, nous analysons la transformation des représentations des Amériques au rythme de coups d'État militaires régionaux. Nous interrogeons le rôle des images dans la représentation des Amériques par une analyse rhétorique, énonciative et pragmatique du langage expérimental qui constitue la marque esthétique du Noticiero ICAIC.

This article develops a semiotic study of the representations of the Americas within the Noticiero ICAIC, Cuban newsreel produced and broadcast between 1960 and 1990. Taking into account the information production conditions in the context of the Cold War, we analyse the transformation of the Americas' representations following the rhythm of regional military Coups d'État. We also interrogate the role of images in the representation of the Americas through a rhetorical, enunciative and pragmatic analysis of the experimental language that constitutes the aesthetic mark of the Noticiero ICAIC.

\section{INDEX}

Keywords : Noticiero ICAIC, newsreel, semiotics, image, representation, experimental language Mots-clés : Noticiero ICAIC, actualités cinématographiques, sémiotique, image, représentation, langage expérimental 
AUTEUR

\section{CAMILA ARÊAS}

Université de La Réunion (LCF)

camila.cabral-areas@univ-reunion.fr 